TRANSACTIONS OF THE

AMERICAN MATHEMATICAL SOCIETY

Volume 355, Number 6, Pages 2557-2568

S 0002-9947(02)03229-4

Article electronically published on December 18, 2002

\title{
TAME SETS, DOMINATING MAPS, AND COMPLEX TORI
}

\author{
GREGERY T. BUZZARD
}

\begin{abstract}
A discrete subset of $\mathbb{C}^{n}$ is said to be tame if there is an automorphism of $\mathbb{C}^{n}$ taking the given discrete subset to a subset of a complex line; such tame sets are known to allow interpolation by automorphisms. We give here a fairly general sufficient condition for a discrete set to be tame. In a related direction, we show that for certain discrete sets in $\mathbb{C}^{n}$ there is an injective holomorphic map from $\mathbb{C}^{n}$ into itself whose image avoids an $\epsilon$-neighborhood of the discrete set. Among other things, this is used to show that, given any complex $n$-torus and any finite set in this torus, there exist an open set containing the finite set and a locally biholomorphic map from $\mathbb{C}^{n}$ into the complement of this open set.
\end{abstract}

\section{INTRODUCTION}

The notion of a tame set was first introduced by Rosay and Rudin in 6]. A discrete set $\Lambda$ of points in $\mathbb{C}^{n}$ is said to be tame if there is an automorphism, $\Phi$, of $\mathbb{C}^{n}$ such that the image of $\Lambda$ under $\Phi$ lies in a complex line. In [2], Buzzard and Forstneric show that, given any two tame sets, there is an automorphism taking one to the other with any prescribed pairing of points and agreeing with any given finite invertible jet at each point of the first tame set. Thus tame sets form a natural class of sets allowing interpolation by automorphisms. However, by [6], it is known that not every discrete set in $\mathbb{C}^{n}$ is tame, and it is not clear in general how to determine if a given discrete set is tame.

In this paper we give a fairly general sufficient condition for a discrete set, $\Lambda$, in $\mathbb{C}^{n}$ to be tame: that there exist a holomorphic projection, $\pi$, from $\mathbb{C}^{n}$ to a complex line (which we may assume is the $z_{1}$-axis) such that $\overline{\pi(\Lambda)}$ is an Arakelian set (see the definition below) and that the interior of $\overline{\pi(\Lambda)}$ has no unbounded components. This follows from the fact that we can find some positive continuous function $f(z)$ on $\overline{\pi(\Lambda)}$ which is constant on each component of the interior $(\overline{\pi(\Lambda)})$ such that the map $\left(z_{1}, z^{\prime}\right) \mapsto\left(z_{1}, z^{\prime} f\left(z_{1}\right)\right)$ takes $\Lambda$ into a cone $\left\{\left(z_{1}, z^{\prime}\right):\left\|z^{\prime}\right\|>\left|z_{1}\right|\right\}$. By a harmonic approximation result similar to Arakelian's theorem, there exists an entire function $g$ whose real part is larger than $\log f$ on $\overline{\pi(\Lambda)}$. Hence the automorphism $\left(z_{1}, z^{\prime}\right) \mapsto\left(z_{1}, z^{\prime} \exp \left(g\left(z_{1}\right)\right)\right)$ takes $\Lambda$ into the same cone. By results of [6], this implies that $\Lambda$ is tame.

In results related to tameness, [6] showed the existence of discrete sets which are unavoidable by nondegenerate holomorphic maps, meaning that, given any holomorphic map of $\mathbb{C}^{n}$ into itself with generic rank $n$, the image of this map must

Received by the editors June 20, 1999.

2000 Mathematics Subject Classification. Primary 32H02; Secondary $32 \mathrm{E} 30$.

Supported in part by an NSF Postdoctoral Fellowship.

(C)2002 American Mathematical Society 
intersect the unavoidable discrete set. From the definition of tame, it is immediate that a tame set is avoidable by holomorphic maps.

Using essentially the same techniques as described above, we show that if $E$ is any subset of $\mathbb{C}^{n}$ such that $\overline{\pi(E)}$ is Arakelian, the interior of $\overline{\pi(E)}$ has no unbounded components, and $E$ does not intersect the $z_{1}$-axis, then $E$ is avoidable by nondegenerate holomorphic maps, and in fact there is a Fatou-Bieberbach domain in the complement of $E$. This allows us to construct examples of Fatou-Bieberbach domains contained in surprisingly small sets.

In a related direction, we define the notion of a discrete set $\Lambda$ in $\mathbb{C}^{n}$ which is $\epsilon$-avoidable by nondegenerate holomorphic maps. By this we mean that there exist some $\epsilon>0$ and some holomorphic map of generic rank $n$ from $\mathbb{C}^{n}$ to itself whose image avoids the union over $p \in \Lambda$ of the balls of radius $\epsilon$ and center $p$. In particular, using the results of the previous paragraph, we show that if $\Lambda$ is a product of a lattice in $\mathbb{C}$ and a lattice in $\mathbb{C}^{n-1}$, then $\Lambda$ is $\epsilon$-avoidable, and even $\epsilon$-avoidable by an injective holomorphic map. Using somewhat more subtle techniques, we show also that if $\pi(\Lambda)$ is contained in a discrete union of certain real curves, and if there is a positive lower bound on the distance between any two of these curves and between any two points in $\Lambda$, then again $\Lambda$ is $\epsilon$-avoidable by an injective holomorphic map.

In particular, taking the curves to be parallel real lines, this implies that the complement of an open set in a complex torus need not be Kobayashi hyperbolic or even measure hyperbolic. More precisely, for any $n$-dimensional complex torus, $T$, and any finite set of points in this torus, there is some open set, $U$, containing this finite set, and a holomorphic map $F: \mathbb{C}^{n} \rightarrow T \backslash U$ with nonvanishing Jacobian determinant. In particular, $F$ is a dominating map: a map from $\mathbb{C}^{n}$ into an $n$ dimensional complex manifold (with boundary) having generic rank $n$. In fact, $F$ lifts to an injective holomorphic map from $\mathbb{C}^{n}$ to $\mathbb{C}^{n}$. This should be compared with [5], in which it was claimed that the complement of any open set in a simple complex torus is Kobayashi hyperbolic (a complex torus is simple if it has no nontrivial complex subtori). There is no contradiction, because it was later realized that the proof given in 5 is incorrect, since the topological closure of a complex oneparameter group need not be a complex torus. Despite this, the validity of the claim was an open question until the current paper, which shows the claim to be false.

I am grateful to the National Science Foundation for support from an NSF Postdoctoral Fellowship, and to l'IHES for its hospitality during the final preparation of this manuscript.

\section{Using Arakelian sets to COnStruct automorphisms}

Following 7], we give the following definition of an Arakelian set in $\mathbb{C}$. First, if $E$ is a closed subset of $\mathbb{C}$, the phrase "hole of $E$ " refers to any bounded component of the complement of $E$.

Definition 2.1. A closed subset $E$ of $\mathbb{C}$ without holes is an Arakelian set if for every closed disk $D$ contained in $\mathbb{C}$, the union of all holes of $E \cup D$ is a bounded set.

This definition is equivalent to the condition that $\hat{\mathbb{C}} \backslash E$ is connected and locally connected, where $\hat{\mathbb{C}}$ is the Riemann sphere. 
Arakelian's theorem, e.g. [7], states that, given any Arakelian set $E$, any complex-valued function, $f$, which is continuous on $E$ and holomorphic in the interior of $E$, and any $\epsilon>0$, there exists an entire function $h$ such that $|h(z)-f(z)|<\epsilon$ for all $z \in E$.

The following proposition will be useful in constructing certain automorphisms.

Proposition 2.2. Let $E$ be an Arakelian subset of $\mathbb{C}$, and suppose that each component of the interior of $E$ is bounded. Let $f$ be continuous and real-valued on $E$. Then there exists an entire function $g$ such that $\operatorname{Re}(g(z)) \geq f(z)$ for all $z \in E$.

Remark 2.3. In fact, the hypotheses can be weakened to require that $\partial \hat{E}=\partial E$ and that $(\mathbb{C}, E)$ satisfies the $(K, L)$ condition. For definitions, see [4. Sections 1.7, 3.7, $3.10]$.

Definition 2.4. For $p \in \mathbb{C}^{n}$ and $r>0, \mathbb{B}(p ; r)$ is the ball of center $p$ and radius $r$.

Proof. For each component $U$ of the interior of $E$, let $M(U)$ be the supremum of $f(z)$ over $z \in \bar{U}$. Let $h_{0}(z)=f(z)$ if $z$ is not in the interior of $E$, and $h_{0}(z)=M(U)$ if $z$ is in the component $U$ of the interior of $E$. Since each component $U$ is bounded, $h_{0}$ is bounded on compact subsets of $E$. Hence there exists a continuous real-valued function $h$ on $E$ with $h(z) \geq h_{0}(z)$ for all $z \in E$ (e.g., define $h_{1}(z)$ on $\mathbb{C}$ to be the supremum of $h_{0}(w)$ over all $w \in E$ with $|w| \leq|z|+1$, then take the convolution of $h_{1}$ with a bump function with support in the disk of radius 1 and restrict to $E$ to obtain $h)$.

Next, let $U$ be a connected component of the interior of $E$, and restrict $h$ to the boundary of $U$. Recall that the Dirichlet problem for $U$ with boundary data $h$ is to construct a function harmonic on $U$ which extends continuously to $\bar{U}$ and which equals $h$ on $\partial U$. For background on the Dirichlet problem and the related ideas used in the remainder of the current proof, see 4. We claim that the Dirichlet problem is solvable on $U$. To prove this, it suffices to show that each point $z \in \partial U$ is a regular point for $U$. Since $U$ is bounded; hence Greenian, [4, Theorem 0.G] implies that it suffices to show that $\mathbb{C} \backslash U$ is not thin at $z$. By [4, Theorem 0.D], if $\mathbb{C} \backslash U$ is thin at $z$, then there are arbitrarily small positive values of $r$ such that $\partial \mathbb{B}(z ; r) \subset U$. Since $z \in \partial U$, this implies that the connected component, $A$, of $\mathbb{C} \backslash U$ containing $z$ is bounded. But $A$ contains the connected component of $\mathbb{C} \backslash E$ containing $z$, and since $E$ is Arakelian, this latter component is unbounded; hence $A$ is unbounded. Thus $\mathbb{C} \backslash U$ cannot be thin at $z$. So $U$ is a regular set for the Dirichlet problem, and hence $u$ extends continuously to $h$ on $\partial U$. Moreover, since $h(z) \geq M(U)$ for all $z \in \partial U$, we have by the minimum principle for harmonic functions that $u(z) \geq M(U) \geq f(z)$ for all $z \in U$.

Solving the Dirichlet problem with boundary data $h$ on each component of the interior of $E$ and extending to $\partial E$ by $u=h$ gives a function $u$ which is continuous on $E$, harmonic in the interior of $E$, and equal to $h$ on the boundary of $E$. Since $E$ is Arakelian, it satisfies the conditions in part (c) of [4 Corollary 3.21], and hence there exists a function $v$ harmonic on $\mathbb{C}$ such that $0<v-u<1$ on $E$. Since $v$ is harmonic on $\mathbb{C}$, there exists an entire $g$ with $\operatorname{Re}(g(z))=v(z)$. Then for all $z \in E$, $\operatorname{Re}(g(z)) \geq u(z) \geq f(z)$, as desired.

Using Arakelian's theorem, we prove the following theorem, which states roughly that any closed set in $\mathbb{C}^{n}$ which avoids the $z_{1}$-axis and which projects to an Arakelian set in the $z_{1}$-axis as in proposition 2.2 can be pushed by an automorphism to lie 
in a kind of conical region in which $z_{1}$ is the smallest coordinate in absolute value. For notation, $p=\left(p^{1}, \ldots, p^{n}\right)$ is a point in $\mathbb{C}^{n}, \pi(p)=p^{1}$ is projection to the first coordinate (the $z_{1}$-axis), and $\pi^{\prime}$ is projection to the remaining $n-1$ coordinates. Also, $p^{\prime}=\pi^{\prime}(p)$, and a set $E \subset \mathbb{C}^{n}$ does not intersect the $z_{1}$-axis provided that $\left\|\pi^{\prime}(p)\right\| \neq 0$ whenever $p \in E$. Note that the hypotheses of the following theorem can be weakened as in the remark after proposition 2.2

Theorem 2.5. Suppose that $E$ is a closed subset of $\mathbb{C}^{n}$ that does not intersect the $z_{1}$-axis, that $\overline{\pi(E)}$ is an Arakelian set, and that each component of the interior of $\overline{\pi(E)}$ is bounded. Then there exists an automorphism of $\mathbb{C}^{n}$ of the form $F(p)=$ $\left(\pi(p), \exp (g(\pi(p))) p^{\prime}\right)$ such that

$$
F(E) \subset\left\{p:\left\|p^{\prime}\right\| \geq|\pi(p)|+1\right\} .
$$

Moreover, there is an injective holomorphic map from $\mathbb{C}^{n}$ into the complement of E.

The conclusion of this theorem relates to [6, Theorem 3.5], which states as a special case that if $\Lambda$ is a discrete set in $\mathbb{C}^{n}$ such that $\pi^{\prime}(\Lambda)$ is discrete in $\mathbb{C}^{n-1}$ and if for each $q^{\prime} \in \pi^{\prime}(\Lambda)$ there are only finitely many $p \in \Lambda$ satisfying $\pi^{\prime}(p)=q^{\prime}$, then $\Lambda$ is tame. In particular, any discrete set contained in the right-hand set in (2.1) satisfies this condition, hence is tame. Thus we obtain the following.

Corollary 2.6. Let $\Lambda \subset \mathbb{C}^{n}$ be discrete such that $\overline{\pi(\Lambda)}$ is Arakelian and the interior of $\overline{\pi(\Lambda)}$ has no unbounded components. Then $\Lambda$ is tame.

Proof of Theorem [2.5. Let $E_{1}=\overline{\pi(E)}$, and note that $E_{1}$ satisfies the hypotheses of proposition 2.2 For $z \in E_{1}$, let $f(z)$ be the distance from the point $(z, 0, \ldots, 0)$ to $E$. Since $E$ is closed and does not intersect the $z_{1}$-axis, $f$ is positive and continuous on $E_{1}$. Hence by proposition 2.2, there exists an entire function $g$ such that if $z \in E_{1}$, then $\operatorname{Re}(g(z)) \geq \log ((1+|z|) / f(z))$.

Define $F(p)=\left(\pi(p), \exp (g(\pi(p))) p^{\prime}\right)$, in which case $F$ is an automorphism of $\mathbb{C}^{n}$. Suppose that $p \in E$. Then $\left\|p^{\prime}\right\| \geq f(\pi(p))$; so $\operatorname{Re}(g(\pi(p))) \geq \log \left((1+|\pi(p)|) /\left\|p^{\prime}\right\|\right)$. Hence $\left\|\exp (g(\pi(p))) p^{\prime}\right\| \geq 1+|\pi(p)|$, which proves (2.1).

To finish the proof of the theorem, we use the fact that the basin of attraction of the fixed point of an automorphism of $\mathbb{C}^{n}$ is biholomorphic to $\mathbb{C}^{n}$; i.e., it is a Fatou-Bieberbach domain (see, e.g., [6, appendix]). Let

$$
H(p)=\left(p^{2}, \ldots, p^{n},\left(\left(p^{2}\right)^{2}-p^{1}\right) / 2\right),
$$

and let

$$
G(q)=H^{-1}(q)=\left(\left(q^{1}\right)^{2}-2 q^{n}, q^{1}, \ldots, q^{n-1}\right) .
$$

The origin is a fixed point for $H$ (and $G$ ). Calculating the derivative gives

$$
\left(D_{0} H\right)\left(v^{1}, \ldots, v^{n}\right)^{T}=\left(v^{2}, \ldots, v^{n},-v^{1} / 2\right)^{T} .
$$

Hence an eigenvalue $\lambda$ must satisfy $v^{2}=\lambda v^{1}, \ldots, v^{n}=\lambda v^{n-1}$, and $-v^{1} / 2=\lambda v^{n}$. Hence $-v^{1} / 2=\lambda^{n} v^{1}$, and so the eigenvalues are the $n$th roots of $-1 / 2$. In particular, the origin is an attracting fixed point for $H$; so, as mentioned above, the basin of attraction $D=\left\{p: H^{m}(p) \rightarrow 0\right.$ as $\left.m \rightarrow \infty\right\}$ is a Fatou-Bieberbach domain.

Since $G=H^{-1}$, it follows that for any $R>0, D \subset \bigcup_{m>0} H^{m}\left(\Delta^{n}(0 ; R)\right)$. By [1] , if $R$ is sufficiently large, then $D$ is contained in $\Delta^{n}(0 ; R) \cup\left\{p:|\pi(p)| \geq R,\left\|p^{\prime}\right\| \leq\right.$ $|\pi(p)|\}$. Hence, scaling $D$ by a factor of $1 / R, D$ lies in the complement of $F(E)$. So 
$F^{-1}(D)$ is a Fatou-Bieberbach domain in the complement of $E$, and hence there is an injective holomorphic map from $\mathbb{C}^{n}$ to the complement of $E$.

Remark 2.7. Let

$$
V=\left\{p:\left\|p^{\prime}\right\| \geq 1+|\pi(p)|\right\} .
$$

The preceding proof shows that there is a Fatou-Bieberbach domain contained in the complement of $V$. In [3], this Fatou-Bieberbach domain is used to show that for any algebraic variety of codimension 2 in $\mathbb{C}^{n}$ there is a Fatou-Bieberbach domain in the complement of the variety, hence a dominating map into the complement.

Example 2.8. As an example of a Fatou-Bieberbach domain whose shape is constricted in some sense, let $S_{j}$ be any collection of disjoint, compact sets in the plane, let $\epsilon_{j}>0$ for all $j$, and in $\mathbb{C}^{n}$ let

$$
E=\bigcup_{j \geq 1}\left\{p: \pi(p) \in S_{j},\left\|p^{\prime}\right\| \geq \epsilon_{j}\right\} .
$$

Then $E$ satisfies the hypotheses of theorem 2.5, hence the complement of $E$ contains a Fatou-Bieberbach domain. In particular, taking the $S_{j}$ 's to be squares in the plane, they can be chosen to fill up all but a finite amount of area in the plane, in which case the Fatou-Bieberbach domain is contained in a small neighborhood of the $z_{1}$-axis plus the small "corridors" lying above the complement of the $S_{j}$ 's.

For other examples of Fatou-Bieberbach domains contained in sets which are small in some sense, see [8].

\section{Approximation on a neighborhood of a CURVE}

In section 4 we will discuss sets which are $\epsilon$-avoidable by nondegenerate holomorphic maps. By this we mean a set $E$ such that there exist some $\epsilon>0$ and a holomorphic map $F: \mathbb{C}^{n} \rightarrow \mathbb{C}^{n}$ of generic rank $n$ such that $\operatorname{dist}\left(F\left(\mathbb{C}^{n}\right), E\right) \geq \epsilon$. We first require some approximation results in one variable. In the current section, $x$ and $y$ are always real.

Definition 3.1. For $\epsilon_{x}, \epsilon_{y}>0$, let $S\left(\epsilon_{y}\right)=\left\{x+i y:|y|<\epsilon_{y}\right\}$, and let $T\left(\epsilon_{x}\right)=$ $\left\{x+i y:|x|>\epsilon_{x},|y|<|x|-\epsilon_{x}\right\}$.

Lemma 3.2. Let $\epsilon_{x}, \epsilon_{y}>0$. Then there exists $g$ holomorphic on $S\left(\epsilon_{y}\right) \cup T\left(\epsilon_{x}\right)$ such that

(1) $|g(z)-1|<\frac{4 \epsilon_{y}}{\pi \epsilon_{x}}$ if $z=x+i y,|x|<\frac{\epsilon_{x}}{2},|y|<\epsilon_{y}$.

(2) $\operatorname{Re}(g)>0$ and $|\operatorname{Im}(g)| \leq 2 \operatorname{Re}(g)$ on $S\left(\epsilon_{y}\right) \cup T\left(\epsilon_{x}\right)$.

(3) If $z \in T\left(\epsilon_{x}\right)$, then

$$
|g(z)| \leq\left(\frac{\epsilon_{x} \epsilon_{y}}{\left(|z|-\epsilon_{x}\right)^{2}}\right)^{2 / 3}
$$

Proof. Let

$$
\begin{aligned}
h(z) & =\frac{1}{2 \pi i} \int_{-\epsilon_{x}}^{\epsilon_{x}}\left(\frac{1}{t-i \epsilon_{y}-z}-\frac{1}{t+i \epsilon_{y}-z}\right) d t \\
& =\frac{1}{\pi} \int_{-\epsilon_{x}}^{\epsilon_{x}} \frac{\epsilon_{y}}{(t-z)^{2}+\epsilon_{y}^{2}} d t .
\end{aligned}
$$


Then $h$ is holomorphic except on the set $\left\{x \pm i \epsilon_{y}:-\epsilon_{x} \leq x \leq \epsilon_{x}\right\}$, hence holomorphic on $S\left(\epsilon_{y}\right) \cup T\left(\epsilon_{x}\right)$. Also, contour integration shows that the second integral evaluated over the entire real line is 1 whenever $|\operatorname{Im} z| \neq \epsilon_{y}$.

We will define $g(z)=h(z)^{2 / 3}$. Note that for $t$ real, we have

$$
\operatorname{Re}\left((t-z)^{2}+\epsilon_{y}^{2}\right)=(t-x)^{2}-y^{2}+\epsilon_{y}^{2}
$$

which is positive if $z \in S\left(\epsilon_{y}\right) \cup T\left(\epsilon_{x}\right)$. Hence $\operatorname{Re}(h(z))>0$. So $g$ can be defined as above, and the root can be chosen so that $\arg (g(z)) \in(-\pi / 3, \pi / 3)$. Thus part 2 of the lemma holds.

For part 1 of the lemma, let $z=x+i y$ with $|x|<\epsilon_{x} / 2$ and $|y|<\epsilon_{y}$. Then

$$
\begin{aligned}
|h(z)-1| & =\frac{1}{\pi}\left|\left(\int_{-\epsilon_{x}}^{\epsilon_{x}}-\int_{-\infty}^{\infty}\right) \frac{\epsilon_{y}}{(t-z)^{2}+\epsilon_{y}^{2}} d t\right| \\
& \leq \frac{\epsilon_{y}}{\pi} \int_{|t| \geq \epsilon_{x}} \frac{1}{(t-x)^{2}} d t \\
& \leq\left(\frac{2 \epsilon_{y}}{\pi}\right) \frac{1}{\epsilon_{x}-|x|} .
\end{aligned}
$$

Since $|x|<\epsilon_{x} / 2$ by assumption, and since $|g(z)-1| \leq|h(z)-1|$, this gives part 1 .

For part 3 , if $z=x+i y \in T$, then a simple computation shows that

$$
\left|(t-z)^{2}+\epsilon_{y}^{2}\right| \geq(t-x)^{2}+y^{2} .
$$

Hence

$$
|h(z)| \leq \frac{\epsilon_{y}}{\pi} \int_{-\epsilon_{x}}^{\epsilon_{x}} \frac{d t}{(t-x)^{2}+y^{2}},
$$

which immediately implies part 3.

The following lemma gives conditions under which the graph of a real-valued function is contained in the union of sets $S$ and $T$ from the previous lemma. As usual, $\|f\|_{\infty}$ refers to the $L^{\infty}$-norm of a function.

Definition 3.3. For a set $\Gamma \subset \mathbb{C}$, let $\Gamma_{\epsilon}=\{z: \operatorname{dist}(z, \Gamma) \leq \epsilon\}$.

Lemma 3.4. Let $f: \mathbb{R} \rightarrow \mathbb{R}$ be $C^{2}$ with $\left\|f^{\prime}\right\|_{\infty}<\sqrt{2}-1$ and $\left\|f^{\prime \prime}\right\|_{\infty}<\infty$. Let $\gamma(x)=x+i f(x)$ and write $\gamma^{\prime}(0)=r e^{i \theta}, \theta \in(-\pi / 2, \pi / 2)$. Then $\Gamma=e^{-i \theta} \gamma(\mathbb{R})$ is the graph of a function $h: \mathbb{R} \rightarrow \mathbb{R}$ such that $h(0)=h^{\prime}(0)=0,\left\|h^{\prime}\right\|_{\infty}<1$, and $\left\|h^{\prime \prime}\right\|_{\infty}<K\left\|f^{\prime \prime}\right\|_{\infty}$ for some $K$ independent of $f$. Moreover, if $A=\left\|h^{\prime \prime}\right\|_{\infty}$ and $\epsilon<1 /(12 A)$, then $\Gamma_{\epsilon} \subset S(2 \epsilon) \cup T(\sqrt{\epsilon / 2 A})$.

Proof. Since $\sqrt{2}-1=\tan (\pi / 8)$, it follows that $|\theta|<\pi / 8$. Let $\tau(x)=e^{-i \theta} \gamma(x)$ and let $\tau_{1}(x)=\operatorname{Re}(\tau(x))$ and $\tau_{2}(x)=\operatorname{Im}(\tau(x))$. Then $\tau_{1}^{\prime}(x)=\cos \theta+f^{\prime}(x) \sin \theta$. So $\left\|\tau_{1}^{\prime}\right\|_{\infty}<\infty$, and the bounds on $\theta$ and $\left\|f^{\prime}\right\|_{\infty}$ imply that there exists $a>0$, independent of $f$, such that $\tau_{1}^{\prime}(x)>a$.

Hence $\tau_{1}: \mathbb{R} \rightarrow \mathbb{R}$ is bijective; so we can define $\alpha(t)=\tau_{1}^{-1}(t)$ and $h(t)=\tau_{2}(\alpha(\tau))$. Substitution shows that $e^{-i \theta} \gamma(\mathbb{R})$ is the graph of $h$. Moreover, since $f(0)=0$, we have $\tau_{1}(0)=\tau_{2}(0)=0$; hence $h(0)=0$, and

$$
h^{\prime}(t)=\tau_{2}^{\prime}(\alpha(t)) \alpha^{\prime}(t)=\frac{\tau_{2}^{\prime}(\alpha(t))}{\tau_{1}^{\prime}(\alpha(t))} .
$$

Thus $h^{\prime}(t)$ is the tangent of the angle between the real axis and the line tangent to $\tau(\mathbb{R})$ at the point $\tau(\alpha(t))$. By choice of $f$, the absolute value of this angle is at most $|\theta+\pi / 8|<\pi / 4$. Hence $\left\|h^{\prime}\right\|_{\infty}<1$. 
Next, note that $\tau_{1}^{\prime \prime}(x)=f^{\prime \prime}(x) \sin \theta, \tau_{2}^{\prime \prime}(x)=f^{\prime \prime}(x) \cos \theta, \alpha^{\prime}(t)=1 / \tau_{1}^{\prime}(\alpha(t))$, and

Hence

$$
\alpha^{\prime \prime}(t)=-\frac{\tau_{1}^{\prime \prime}(\alpha(t))}{\left(\tau_{1}^{\prime}(\alpha(t))\right)^{2} \tau_{1}^{\prime}(\alpha(t))}
$$

$$
\begin{aligned}
\left|h^{\prime \prime}(t)\right| & =\left|\tau_{2}^{\prime \prime}(\alpha(t))\left(\alpha^{\prime}(t)\right)^{2}+\tau_{2}^{\prime}(\alpha(t)) \alpha^{\prime \prime}(t)\right| \\
& \leq \frac{\left\|f^{\prime \prime}\right\|}{a^{2}}+\frac{\left\|f^{\prime \prime}\right\|}{a^{2}},
\end{aligned}
$$

where $a$ is the lower bound on $\tau_{1}^{\prime}$ as before. This gives the desired upper bound on $\left\|h^{\prime \prime}\right\|_{\infty}$.

To finish the lemma, let $A=\left\|h^{\prime \prime}\right\|_{\infty}$. Since $h(0)=h^{\prime}(0)=0$, we have $|h(x)| \leq$ $A|x|^{2} / 2$ for all $x$. Let $\epsilon<1 /(12 A)$, let $\epsilon_{y}=2 \epsilon$, and let $\epsilon_{x}=\sqrt{\epsilon /(2 A)}$.

If $|x| \leq 2 \epsilon_{x}$, then $|h(x)| \leq 4 A \epsilon_{x}^{2} / 2=\epsilon$; so $\overline{\mathbb{B}(x+i h(x), \epsilon)} \subset S\left(\epsilon_{y}\right)$. If $x>2 \epsilon_{x}$, then

$$
|h(x)|=\left|\int_{2 \epsilon_{x}}^{x} h^{\prime}(t) d t+h\left(2 \epsilon_{x}\right)\right|<|x|-2 \epsilon_{x}+\epsilon,
$$

with the same final bound if $x<-2 \epsilon_{x}$. Moreover, for $z=x+i y \in T\left(\epsilon_{x}\right)$, we have $\operatorname{dist}(z, \mathbb{C} \backslash T)=\left(|x|-\epsilon_{x}-|y|\right) / \sqrt{2}$. So for $\overline{\mathbb{B}(x+i h(x), \epsilon)} \subset T\left(\epsilon_{x}\right)$, we need $\left(|x|-\epsilon_{x}-|h(x)|\right)>\epsilon \sqrt{2}$, or $\epsilon_{x}>\epsilon(1+\sqrt{2})$, which is satisfied for $\epsilon<1 /(12 A)$.

Hence for all $\epsilon<1 /(12 A), \Gamma_{\epsilon} \subset S(2 \epsilon) \cup T(\sqrt{\epsilon /(2 A)})$, as desired.

The following lemma implies that for certain well-behaved curves in the plane, there exists a function holomorphic in an $\epsilon$-neighborhood of the curve whose real part is positive and which is very near 0 in an $\epsilon$-neighborhood of certain prescribed points and whose real part is large far away from all of these prescribed points. The function constructed here will later be approximated and exponentiated to construct an automorphism.

Lemma 3.5. Suppose $f: \mathbb{R} \rightarrow \mathbb{R}$ is $C^{2}$ with $\left\|f^{\prime}\right\|_{\infty}<\sqrt{2}-1$ and $\left\|f^{\prime \prime}\right\|_{\infty}<\infty$. Let $\Gamma=\{x+i f(x): x \in \mathbb{R}\}$, and let $\left\{z_{j}\right\}_{j=-M}^{N} \subset \Gamma$ be such that $\left|z_{j}-z_{k}\right| \geq 1$ if $j \neq k$ (here one or both of $M, N$ may be $\infty$ ). Let $K>1$. Then for all $\epsilon$ sufficiently small, depending only on $\left\|f^{\prime \prime}\right\|$ and $K$, there exists a function $g$, holomorphic on $\Gamma_{\epsilon}$, such that

(1) $\operatorname{Re}(g)>0$ on $\Gamma_{\epsilon}$;

(2) $|g(z)|<\frac{1}{K}$ if $\left|z-z_{j}\right|<\epsilon$ for some $j$;

(3) $\operatorname{Re}(g(z)) \geq K$ if $\left|z-z_{j}\right|>\frac{1}{2}$ for all $j$.

Remark 3.6. In part 3 , the constant $1 / 2$ can be replaced by $C \sqrt{\epsilon}$, where $C$ is a constant depending only on $\left\|f^{\prime \prime}\right\|$.

Proof. Fix $z_{j}$. By translation, we may assume $z_{j}=0(f(0)=0)$. Write $r e^{i \theta}=$ $1+i f^{\prime}(0)$, where $|\theta|<\pi / 8$. By the previous lemma, there exist constants $C>0$ and $\epsilon_{0}>0$, depending only on $\left\|f^{\prime \prime}\right\|_{\infty}$, such that if $0<\epsilon<\epsilon_{0}$, then $e^{-i \theta} \Gamma_{\epsilon} \subset S(2 \epsilon) \cup$ $T(C \sqrt{\epsilon})$. For the moment, choose $\epsilon \in\left(0, \epsilon_{0}\right)$ small enough that $C \sqrt{\epsilon}>2 \epsilon$, although we will shrink $\epsilon$ later as needed. By lemma 3.2 there exists $h_{j}$, holomorphic on $S(2 \epsilon) \cup T(C \sqrt{\epsilon})$, such that

(1) $\left|h_{j}(z)-1\right|<\frac{8 \sqrt{\epsilon}}{C \pi}$ if $z=x+i y,|x|<\frac{C \sqrt{\epsilon}}{2},|y|<2 \epsilon$; 
(2) $\operatorname{Re}\left(h_{j}\right)>0$ and $\left|\operatorname{Im}\left(h_{j}\right)\right| \leq 2 \operatorname{Re}\left(h_{j}\right)$ on $S(2 \epsilon) \cup T(C \sqrt{\epsilon})$;

(3) if $z \in T(C \sqrt{\epsilon})$, then

$$
\left|h_{j}(z)\right| \leq \frac{(2 C)^{2 / 3} \epsilon}{(|z|-C \sqrt{\epsilon})^{4 / 3}} .
$$

Define $g_{j}(z)=h_{j}\left(e^{-i \theta} z\right)$, so that $g_{j}$ is holomorphic on $\Gamma_{\epsilon}$. Moreover, $\operatorname{Re}\left(g_{j}\right)>0$ and $\left|\operatorname{Im}\left(g_{j}\right)\right| \leq 2 \operatorname{Re}\left(g_{j}\right)$ on $\Gamma_{\epsilon}$, and since $C \sqrt{\epsilon} / 2>\epsilon$, we have $\left|g_{j}(z)-1\right|<8 \sqrt{\epsilon} /(C \pi)$ if $\left|z-z_{j}\right|<\epsilon$. Also, if $z \in \Gamma_{\epsilon}$ with $\left|z-z_{j}\right|>C \sqrt{\epsilon}+2 \epsilon$, then

$$
\left|g_{j}(z)\right| \leq \frac{(2 C)^{2 / 3} \epsilon}{\left(\left|z-z_{j}\right|-C \sqrt{\epsilon}\right)^{4 / 3}} .
$$

Without loss, we may assume that $z_{j}=x_{j}+i f\left(x_{j}\right)$ with $x_{j}<x_{j+1}$ for all $j$. Since $\left|z_{j}-z_{j+1}\right| \geq 1$ by assumption, and since $\left\|f^{\prime}\right\|_{\infty}<\sqrt{2}-1$, we have $\left|x_{j}-x_{j+1}\right| \geq 1 / 2$ for all $j$. Hence $\left|z_{j}-z_{k}\right| \geq|j-k| / 2$ for all $j, k$.

Define $\hat{g}(z)=2 K \sum_{j=-M}^{N} g_{j}(z)$. The bound in (3.2) together with $\left|z_{j}-z_{k}\right| \geq$ $|j-k| / 2$ implies that this series converges uniformly on compact subsets of $\Gamma_{\epsilon}$; so $\hat{g}$ is holomorphic on $\Gamma_{\epsilon}$. Moreover, $\operatorname{Re}(\hat{g})>0$ and $|\operatorname{Im}(\hat{g})| \leq 2 \operatorname{Re}(\hat{g})$ on $\Gamma_{\epsilon}$, since this is true of each $g_{j}$. Also, if $\left|z-z_{j}\right|>C \sqrt{\epsilon}+2 \epsilon$ for all $j$, then (3.2) together with the fact that $\left|z-z_{n}\right|$ is bounded below by a constant times $|n|$ implies that $|\hat{g}(z)| \leq K_{1} \epsilon$ for some constant $K_{1}$ independent of $z$ subject to $\left|z-z_{j}\right|>C \sqrt{\epsilon}+2 \epsilon$ for all $j$.

Next, suppose $\left|z-z_{j}\right|<\epsilon$ for some $j$. Then

$$
\begin{aligned}
|\hat{g}(z)-2 K| & \leq 2 K\left|g_{j}(z)-1\right|+2 K \sum_{n \neq j}\left|g_{j}(z)\right| \\
& \leq \frac{16 K \sqrt{\epsilon}}{C \pi}+2 K \epsilon \sum_{n \neq j} \frac{(2 C)^{2 / 3}}{\left(\left|z-z_{n}\right|-C \sqrt{\epsilon}\right)^{4 / 3}} .
\end{aligned}
$$

Since $\left|z-z_{n}\right| \geq(|n-j| / 2)-\epsilon$, this last expression is bounded above by $K_{2} \sqrt{\epsilon}$ for some $K_{2}>0$ independent of $j$ and independent of $z$ subject to $\left|z-z_{j}\right|<\epsilon$.

Finally, let $g(z)=1 / \hat{g}(z)$. Then $g$ is holomorphic on $\Gamma_{\epsilon}$, and the properties of $\hat{g}$ imply immediately that for sufficiently small $\epsilon$ depending only on $\left\|f^{\prime \prime}\right\|_{\infty}$ and $K$, all of the conclusions of the lemma are satisfied.

\section{Avoiding An $\epsilon$-NeighBORHOOD}

As before, for $E \subset \mathbb{C}^{n}$, let $E_{\epsilon}=\{z: \operatorname{dist}(z, E) \leq \epsilon\}$.

Definition 4.1. A set $E \subset \mathbb{C}^{n}$ is $\epsilon$-avoidable if there exist $\epsilon>0$ and a holomorphic map $F: \mathbb{C}^{n} \rightarrow \mathbb{C}^{n} \backslash E_{\epsilon}$ such that the generic rank of $F$ is $n$.

By the results of section 2, it follows that if there exists $\epsilon>0$ such that $E_{\epsilon}$ avoids a complex line $L$ and if the projection of $E_{\epsilon}$ to $L$ is Arakelian such that the interior of this projection has no unbounded components, then $E$ is $\epsilon$-avoidable. In particular, if $\Lambda_{1}$ is a lattice of points in $\mathbb{C}$ and $\Lambda^{\prime}$ is a lattice of points in $\mathbb{C}^{n-1}$, then $E=\Lambda_{1} \times \Lambda^{\prime}$ is $\epsilon$-avoidable. Here, a lattice is the span over $\mathbb{Z}$ of $2 n \mathbb{R}$-linearly independent vectors in $\mathbb{C}^{n}$.

Recall that the Hopf surface is defined as the quotient of $\mathbb{C}^{2} \backslash\{0\}$ modulo the equivalence relation $z \equiv 2 z$. The techniques of section 2 can also be used to show that any finite set of points in the Hopf surface is $\epsilon$-avoidable. This can be done by lifting to $\mathbb{C}^{2}$ and choosing coordinates so that no two lifted points project to the 
same point on the $z_{1}$-axis. Sufficiently small balls around each point in the Hopf surface will lift to balls which again have disjoint projections to the $z_{1}$-axis, and this set of lifted balls can be avoided as in the example after corollary [2.6

However, for a general lattice in $\mathbb{C}^{n}$, any projection to a complex line may be dense in some real line. Hence the argument above is not sufficient to show that such a lattice is $\epsilon$-avoidable. Using the techniques of approximation from the previous section, we prove the following theorem which gives sufficient conditions for a discrete set in $\mathbb{C}^{n}$ to be $\epsilon$-avoidable. An immediate corollary of this theorem is that a general lattice in $\mathbb{C}^{n}$ is $\epsilon$-avoidable.

For future reference, $\Delta^{n}(p ; r)$ denotes the polydisk with center $p$ and radius $r$ in each coordinate direction, $\|\cdot\|$ is the standard Euclidean norm on $\mathbb{C}^{n}$, and for $p=\left(p^{1}, \ldots, p^{n}\right) \in \mathbb{C}^{n},\|p\|_{\infty}=\max \left\{\left|p^{j}\right|, j=1, \ldots, n\right\}$.

Theorem 4.2. Let $\Lambda \subset \mathbb{C}^{n}$ be discrete, and let $\pi$ be projection to the first coordinate. Suppose that $\pi(\Lambda)$ is contained in $\bigcup_{m} \Gamma_{m}$, where each $\Gamma_{m}$ is the graph of a $C^{2}$ function $f_{m}: \mathbb{R} \rightarrow \mathbb{R}$ with $\left\|f_{m}^{\prime}\right\|_{\infty}<\sqrt{2}-1$ and $\left\|f_{m}^{\prime \prime}\right\|_{\infty} \leq K<\infty$ for some $K$ independent of $m$. Suppose also that $\operatorname{dist}\left(\Gamma_{m}, \Gamma_{l}\right) \geq 2$ if $m \neq l$ and $\|p-q\|_{\infty} \geq 2$ if $p, q \in \Lambda$ with $p \neq q$. Then there exist $\epsilon>0$ and an injective holomorphic map

$$
F: \mathbb{C}^{n} \rightarrow \mathbb{C}^{n} \backslash \Lambda_{\epsilon}
$$

\section{Hence $\Lambda$ is $\epsilon$-avoidable.}

For calculational convenience, theorem 4.2 will be proved by constructing an injective holomorphic $F: \mathbb{C}^{n} \rightarrow \mathbb{C}^{n} \backslash\left(\bigcup_{p \in \Lambda} \Delta^{n}(p ; \epsilon)\right)$.

Proof. We will construct two automorphisms of $\mathbb{C}^{n}$. The first, $G$, will be chosen so that $G\left(\Lambda_{\epsilon}\right)$ has positive distance from some complex line parallel to the $z_{1^{-}}$ axis. This will be achieved by using the approximation results of the previous section together with Arakelian's theorem to obtain an automorphism which pushes points in $\Lambda_{\epsilon}$ which are relatively far away from the $z_{1}$-axis even farther away, while leaving points in $\Lambda_{\epsilon}$ which are near the $z_{1}$-axis essentially unchanged. The second automorphism will be constructed similarly to push the points in $G\left(\Lambda_{\epsilon}\right)$ into the set $V$ of (2.2).

Let $\left\{q_{j}\right\}_{j=1}^{\infty}$ be an enumeration of the set $\left\{q \in \Lambda:\left\|\pi^{\prime} q\right\|_{\infty} \leq 1 / 2\right\}$. Since $\|p-q\|_{\infty} \geq 2$ for all $p, q \in \Lambda$, we see that $\left|\pi\left(q_{j}\right)-\pi\left(q_{k}\right)\right| \geq 2$ if $j \neq k$. Hence lemma 3.5 implies that given $K>1$, there exists $\epsilon_{0}>0$ such that if $\epsilon<\epsilon_{0}$, then for each $m$, there is $g_{m}$, holomorphic on $\left(\Gamma_{m}\right)_{\epsilon}$, such that $\operatorname{Re}\left(g_{m}\right)>0,\left|g_{m}(z)\right|<1 / K$ if $\left|z-\pi q_{j}\right|<\epsilon$ for some $j$, and $\operatorname{Re}\left(g_{m}(z)\right) \geq K$ if $\left|z-\pi q_{j}\right|>1 / 2$ for all $j$.

We show next that the union of all $\left(\Gamma_{m}\right)_{\epsilon}$ is an Arakelian set. Since $\operatorname{dist}\left(\Gamma_{m}, \Gamma_{l}\right) \geq$ 2 if $m \neq l$, the $\left(\Gamma_{m}\right)_{\epsilon}$ are pairwise disjoint for $\epsilon<1$. Since $\Gamma_{m}$ is the graph of $f_{m}$, we see that $\left(\Gamma_{m}\right)_{\epsilon}$ is homeomorphic to the strip $\{x+i y:|y| \leq 1\}$ via a homeomorphism which preserves lines of the form $\operatorname{Re}(z)=$ const. Since the $\left(\Gamma_{m}\right)_{\epsilon}$ are pairwise disjoint, this straightening can be done simultaneously to show that $E=\bigcup_{m}\left(\Gamma_{m}\right)_{\epsilon}$ is homeomorphic to a union of parallel strips in the plane via a homeomorphism which extends to a homeomorphism of $\hat{C}$. Denote this union of strips by $E^{\prime}$. Since $\hat{C} \backslash E^{\prime}$ is connected and locally connected, the same is true of $\hat{C} \backslash E$, and so $E$ is Arakelian.

Hence there exists $g$ entire such that if $z \in\left(\Gamma_{m}\right)_{\epsilon}$, then $\left|g(z)-g_{m}(z)\right|<1 / K$. Define an automorphism $G: \mathbb{C}^{n} \rightarrow \mathbb{C}^{n}$ by

$$
G(p)=\left(\pi(p), p^{\prime} \exp (g(\pi(p)))\right) .
$$


We show next that for large $K$ and small $\epsilon$ there is a complex line in the complement of $G\left(\Lambda_{\epsilon}\right)$. To do this, let $p \in \Lambda_{\epsilon}$ and suppose first that $p \in \Delta^{n}\left(q_{j} ; \epsilon\right)$ for some $q_{j}$. Choose $m$ so that $\pi\left(q_{j}\right) \in \Gamma_{m}$. Then $\left|\pi(p)-\pi\left(q_{j}\right)\right|<\epsilon$; so by the choice of $g_{m}$ and $g$, we have $|g(\pi(p))| \leq 2 / K$. Since $\left\|\pi^{\prime} p\right\|_{\infty}<\epsilon+1 / 2$, we have for $\epsilon<1 / 4$ that

$$
\left\|\pi^{\prime} G(z)\right\|_{\infty} \leq \frac{3}{4} \exp \left(\frac{1}{K}\right) .
$$

For large $K$, this last expression is less than $7 / 8$.

Next, suppose that $p \notin \Delta^{n}\left(q_{j} ; \epsilon\right)$ for any $q_{j}$. Let $q \in \Lambda$ be such that $p \in$ $\Delta^{n}(q ; \epsilon)$, and choose $m$ so that $\pi(q) \in \Gamma_{m}$. If $\left|\pi(q)-\pi\left(q_{j}\right)\right| \leq 1$ for some $j$, then $\left\|\pi^{\prime} q\right\|_{\infty} \geq 3 / 2$, since $\left\|q-q_{j}\right\|_{\infty} \geq 2$ by assumption and since $\left\|\pi^{\prime} q_{j}\right\|_{\infty} \leq 1 / 2$. Hence $\left\|\pi^{\prime} p\right\|_{\infty} \geq(3 / 2)-\epsilon$. Since $\operatorname{Re}(g) \geq-1 / K$ on $\left(\Gamma_{m}\right)_{\epsilon}$, we have

$$
\left\|\pi^{\prime} G(p)\right\|_{\infty} \geq\left(\frac{3}{2}-\epsilon\right) \exp \left(-\frac{1}{K}\right),
$$

which is greater than $5 / 4$ for $K$ large and $\epsilon$ small.

In the remaining case, we have $\left|\pi(q)-\pi\left(q_{j}\right)\right|>1$ for all $j$, and hence we have $\left|\pi(p)-\pi\left(q_{j}\right)\right|>1 / 2$ for $\epsilon<1 / 2$. Thus $|g(\pi(p))| \geq K-1 / K$. Also, since $p \in \Delta^{n}(q ; \epsilon)$ but is not in $\Delta^{n}\left(q_{j} ; \epsilon\right)$ for any $j$, we have $\left\|\pi^{\prime} p\right\|_{\infty} \geq 1 / 2-\epsilon$. Thus

$$
\left\|\pi^{\prime} G(p)\right\|_{\infty} \geq\left(\frac{1}{2}-\epsilon\right) \exp (K-1),
$$

which is greater than 1 for large $K$ and small $\epsilon$.

From (4.1), 4.2), and (4.3), we see that for large $K$ and small $\epsilon$, for every $p \in \Lambda_{\epsilon}$, either $\left\|\pi^{\prime} G(p)\right\|_{\infty} \leq 7 / 8$ or $\left\|\pi^{\prime} G(p)\right\|_{\infty} \geq 5 / 4$. In particular, for such a point $p$ and any $w \in \mathbb{C}$, we have

$$
\|G(p)-(w, 1, \ldots, 1)\|_{\infty} \geq \frac{1}{8} .
$$

Note also that $\pi G(p)=\pi p$ for all $p \in \mathbb{C}^{n}$.

To finish the proof, we will construct $H$ similar to $G$ so that $H\left(G\left(\Lambda_{\epsilon}\right)\right)$ is contained in $V$, where $V$ is as in (2.2). For each $j$, let $z_{j} \in \Gamma_{j}$ and apply lemmas 3.4 and 3.2 to conclude that there exists $h_{j}$, holomorphic on $\left(\Gamma_{j}\right)_{\epsilon}$, such that $\operatorname{Re}\left(h_{j}\right)>0$, $\left|\operatorname{Im}\left(h_{j}\right)\right| \leq 2\left|\operatorname{Re}\left(h_{j}\right)\right|$, and $1 /\left|h_{j}(z)\right| \geq\left|z-z_{j}\right|-1$ (for $\left|z-z_{j}\right|<1$, hence for any $z$ in $\left.\left(\Gamma_{j}\right)_{\epsilon}\right)$. These conditions imply that $\operatorname{Re}\left(1 / h_{j}(z)\right) \geq\left(\left|z-z_{j}\right|-1\right) / 5$ for all $z \in\left(\Gamma_{j}\right)_{\epsilon}$. Since $\Gamma_{\epsilon}$ is an Arakelian set as noted before, there exists $h$ entire which approximates $\left(5 / h_{j}(z)\right)+\left|z_{j}\right|+3$ within distance 1 on each $\left(\Gamma_{j}\right)_{\epsilon}$. Thus $h$ satisfies $\operatorname{Re}(h(z)) \geq|z|+1$ for all $z \in \Gamma_{\epsilon}$. Define

$$
H(p)=\left(\pi(p), 8\left(p^{\prime}-\{1\}^{n-1}\right) \exp (h(\pi(p)))\right) .
$$

Again $H: \mathbb{C}^{n} \rightarrow \mathbb{C}^{n}$ is biholomorphic. Moreover, if $p \in G\left(\Lambda_{\epsilon}\right)$, then

$$
\left\|\pi^{\prime} p-\{1\}^{n-1}\right\|_{\infty} \geq 1 / 8 .
$$

So

$$
\begin{aligned}
\left\|\pi^{\prime} H(p)\right\|_{\infty} & \geq 8\left\|\pi^{\prime} p-\{1\}^{n-1}\right\|_{\infty} \exp (\operatorname{Re}(h(\pi(p)))) \\
& \geq|\pi(p)|+1 .
\end{aligned}
$$

Hence $H G\left(\Lambda_{\epsilon}\right) \subset V$, where $V$ is as in (2.2), and as noted earlier, there exists a biholomorphic map $\Phi: \mathbb{C}^{n} \rightarrow \mathbb{C}^{n} \backslash V$. Taking $F=G^{-1} H^{-1} \Phi$ gives an injective holomorphic map $F: \mathbb{C}^{n} \rightarrow \mathbb{C}^{n} \backslash \Lambda_{\epsilon}$, as desired. 
The following theorem shows that a general lattice in $\mathbb{C}^{n}$ is $\epsilon$-avoidable. It is nearly a direct corollary of the previous theorem.

Theorem 4.3. Let $\Lambda \subseteq \mathbb{C}^{n}$ be a lattice, let $q_{1}, \ldots, q_{m} \in \mathbb{C}^{n}$, let $\Lambda_{0}=\bigcup_{j=1}^{m} \Lambda+q_{j}$. For some $\epsilon>0$ there exists an injective holomorphic map

$$
F: \mathbb{C}^{n} \rightarrow \mathbb{C}^{n} \backslash \bigcup_{q \in \Lambda_{0}} \mathbb{B}^{n}(q ; \epsilon) .
$$

We first give a simple lemma which says that after a suitable linear change of coordinates, $\Lambda_{0}$ projects to a discrete set of parallel real lines in the plane with a lower bound on the distance between any two of these lines and between any two points in $\Lambda_{0}$. The proof is straightforward and perhaps even standard, but is provided for completeness.

Lemma 4.4. There exists an invertible, complex linear transformation $A: \mathbb{C}^{n} \rightarrow$ $\mathbb{C}^{n}$ such that $\operatorname{Im} \pi A\left(\Lambda_{0}\right)$ is a discrete set in $\mathbb{R}$. Moreover, we may assume that if $p, q \in A\left(\Lambda_{0}\right)$ with $p \neq q$, then $|p-q| \geq 2$ and either $\operatorname{Im} \pi p=\operatorname{Im} \pi q$ or $\mid \operatorname{Im} \pi p-$ $\operatorname{Im} \pi q \mid \geq 2$.

Proof. Let $v_{1}, \ldots, v_{2 n}$ be a $\mathbb{Z}$-basis for $\Lambda$, and let $E$ be the span over $\mathbb{R}$ of the first $2 n-1$ of these vectors. Using the real inner product, let $u \neq 0$ be orthogonal to $E$. Using the complex inner product, let $E^{\prime}$ be the $(n-1)$-dimensional complex subspace orthogonal to $u$. Then each vector in $E^{\prime}$ is real orthogonal to $u$; so $E^{\prime} \subseteq E$. Choose $A_{1}$ complex linear such that $\pi A_{1}(u)=1, \pi^{\prime} A_{1}(u)=0$, and $\pi A_{1}\left(E^{\prime}\right)=0$. Then $\pi A_{1}(E)$ is a one (real) dimensional subspace of $\mathbb{C}$; by rotating in the first coordinate, we may assume that $\pi A_{1}(E)$ is the real line in $\mathbb{C}$.

Let $y_{0}=\operatorname{Im} \pi A_{1}\left(v_{2 n}\right)$, and $y_{j}=\operatorname{Im} \pi A_{1}\left(q_{j}\right)$ for $j=1, \ldots, m$. Then for each $j=1, \ldots, m$ and $k \in \mathbb{Z}$, we have

$$
\pi A_{1}\left(E+k v_{2 n}+q_{j}\right) \subseteq \mathbb{R}+i\left(k y_{0}+y_{j}\right)
$$

so that $\operatorname{Im} \pi A_{1}\left(\Lambda_{0}\right)$ is discrete in $\mathbb{R}$. Applying an appropriate dilation to $A_{1}$ gives $A$ as desired.

Proof of Theorem 4.3. Choose an invertible, complex linear $A$ as in lemma 4.4 Without loss of generality, we may replace $\Lambda$ by $A(\Lambda), q_{j}$ by $A\left(q_{j}\right)$, and $\Lambda_{0}$ by $A\left(\Lambda_{0}\right)$. Then $\pi \Lambda_{0}$ is contained in $\bigcup_{k=1}^{\infty} L_{k}$, where each $L_{k}$ is a line of the form $\mathbb{R}+i a_{k}, a_{k}$ real. Moreover, $\operatorname{dist}\left(L_{j}, L_{k}\right) \geq 2$ if $j \neq k$, and $\|p-q\|_{\infty} \geq 2$ if $p, q \in \Lambda_{0}$ with $p \neq q$. Hence theorem 4.2 implies that $\Lambda_{0}$ is $\epsilon$-avoidable, as claimed.

In particular, given any complex $n$-torus and any finite set of points in this torus, this set of points lifts to a set $\Lambda_{0}$ as in theorem 4.3. Since $\Lambda_{0}$ is $\epsilon$-avoidable, this implies that there exist $\epsilon>0$ and a holomorphic map $F$ from $\mathbb{C}^{n}$ into the torus whose image has distance at least $\epsilon$ from the given set of points. In particular, this gives a dominating map into the complement of a union of balls in this complex $n$-torus, as claimed earlier.

\section{References}

1. E. Bedford and V. Pambuccian, Dynamics of shift-like polynomial diffeomorphisms of $\mathbb{C}^{N}$. Conformal Geometry and Dynamics (Electron. J. Amer. Math. Soc.) 2 (1998), 45-55. MR 99e:32042

2. G. Buzzard and F. Forstneric, An interpolation theorem for holomorphic automorphisms of $\mathbb{C}^{n}$. J. Geom. Anal. 10 (2000), 101-108. MR 2001d:32020 
3. G. Buzzard and J. H. Hubbard, A Fatou-Bieberbach domain avoiding a neighborhood of a variety of codimension 2. Math. Ann. 316 (2000), 699-702. MR 2001d:32024

4. S. Gardiner, Harmonic Approximation. London Math. Soc. Lecture Note Series, no. 221, Cambridge University Press, Cambridge, 1995. MR 96j:31001

5. M. Green, Holomorphic maps to complex tori. Amer. J. Math., 100 (1978), no. 3, 615-620. MR 81c:32048

6. J.-P. Rosay and W. Rudin, Holomorphic maps from $\mathbb{C}^{n}$ to $\mathbb{C}^{n}$. Trans. Amer. Math. Soc., 310 (1988), no. 1, 47-86. MR 89d:32058

7. J.-P. Rosay and W. Rudin, Arakelian's approximation theorem. Amer. Math. Monthly, 96 (1989), no. 5, 432-434. MR 90h:30091

8. J.-P. Rosay and W. Rudin, Growth of volume in Fatou-Bieberbach domains. Publ. Res. Inst. Math. Sci. Kyoto Univ., 29 (1993), no. 1, 161-166. MR 93k:32045

Department of Mathematics, Purdue University, West Lafayette, Indiana 47907

E-mail address: buzzard@math.purdue.edu 